\title{
PERFORMANCE OF BUNCH-BY-BUNCH FEEDBACK SYSTEMS AT BESSY-I*
}

\author{
S. Khan ${ }^{\dagger}$ T. Knuth, BESSY, Berlin, Germany
}

\begin{abstract}
Longitudinal and transverse bunch-by-bunch feedback systems have been in routine operation at the BESSY-II storage ring since December 1999. This paper reviews the operational experience, modifications and the present status of both systems.
\end{abstract}

\section{INTRODUCTION}

BESSY II is a high-brilliance synchrotron radiation source in Berlin-Adlershof [1]. Presently, standard user operation is at a beam energy of $1.7 \mathrm{GeV}$ with a beam current of $250 \mathrm{~mA}$ in 360 bunches.

In 1999, bunch-by-bunch feedback systems were installed to counteract longitudinal and transverse multibunch oscillations. The main source of longitudinal instabilities are higher-order modes (HOMs) of four 3rdharmonic cavities, also installed in 1999 and operated in bunch-lengthening mode in order to increase the beam lifetime. Transverse instabilities are believed to be caused by the finite conductivity of the vacuum chamber walls (resitive-wall effect).

Both systems are routinely employed and significantly improve the quality of synchrotron radiation delivered to the users. At the 9th harmonic of the U-49 undulator, the longitudinal feedback (LFB) doubles the photon intensity. The transverse feedback (TFB) reduces the horizontal size of the beam spot by a factor of 1.5 and the vertical size by a factor of 6 or more. Thus, a gain in radiation brilliance of more than an order of magnitude is achieved.

Due to the availability of backup electronics, the few hardware problems that occurred so far, were quickly solved. Otherwise, little retuning or maintainance was required for both systems. Commisioning of the LFB and TFB was described in [2] and [3], respectively.

\section{SYSTEM DESCRIPTION}

\subsection{Longitudinal Feedback System}

The LFB electronics originally developed for PEP-II (Stanford), the ALS (Berkeley) and for DA $\Phi$ NE (Frascati) is used in combination with a kicker cavity, designed for DA $\Phi N E$ [4] and adapted to the BESSY requirements [5]. More details of the electronics and software architecture can be found in [6] and references therein.

As shown in figure 1, the bunch signal from a pickup passes a comb filter to produce a burst for phase detection

\footnotetext{
* Funded by the Bundesministerium für Bildung, Wissenschaft Forschung und Technologie and by the Land Berlin.

† E-mail: shaukat.khan@bessy.de.
}

at $3 \mathrm{GHz}$. The moment signal (phase-charge) is digitized at a rate of $500 \mathrm{MHz}$, downsampled and handed to an array of 40 DPSs, where a 6-tap FIR filter produces a correction signal for every bunch. The D/A-converted output modulates a quadrature phase shift keyed (QPSK) carrier at $1374 \mathrm{MHz}$ (11/4 times the rf frequency). The correction signal is applied to a $220 \mathrm{~W}$ solid-state power amplifier that drives the kicker cavity.

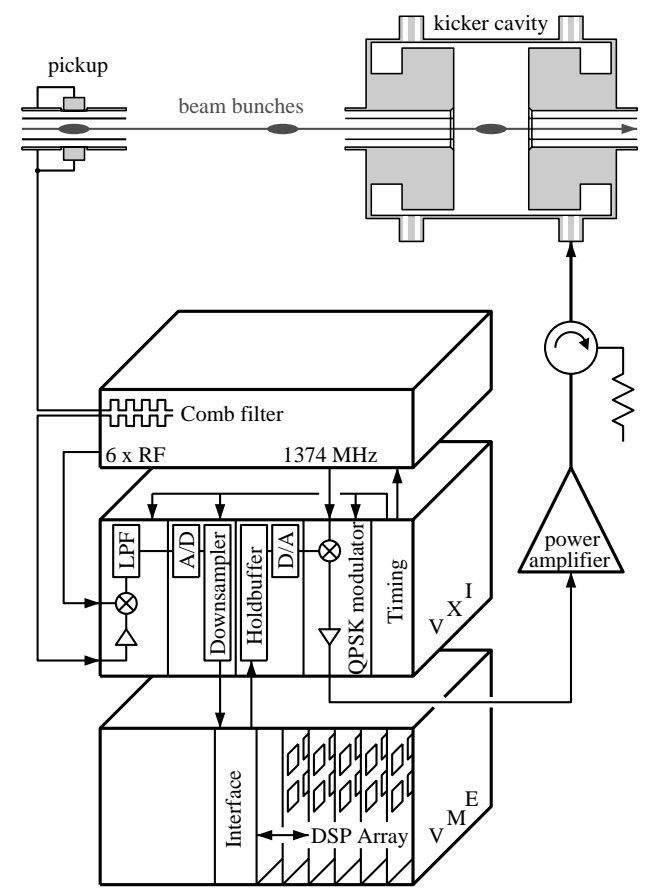

Figure 1: Layout of the longitudinal feedback system.

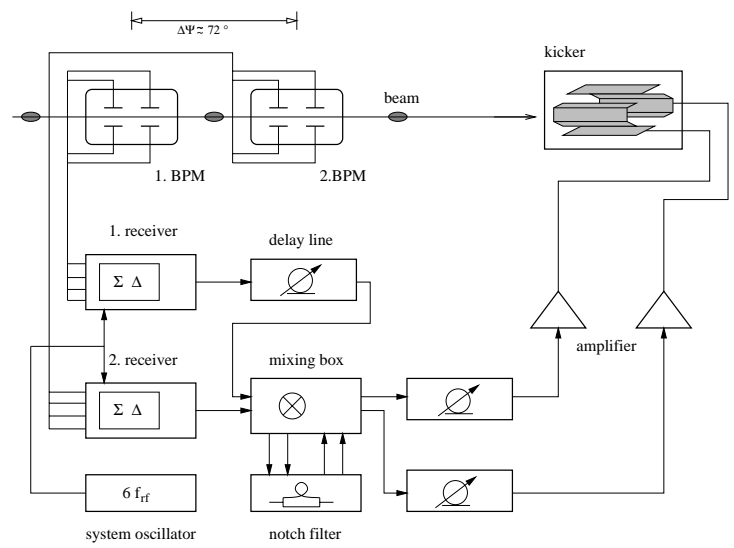

Figure 2: Layout of the transverse feedback system. 


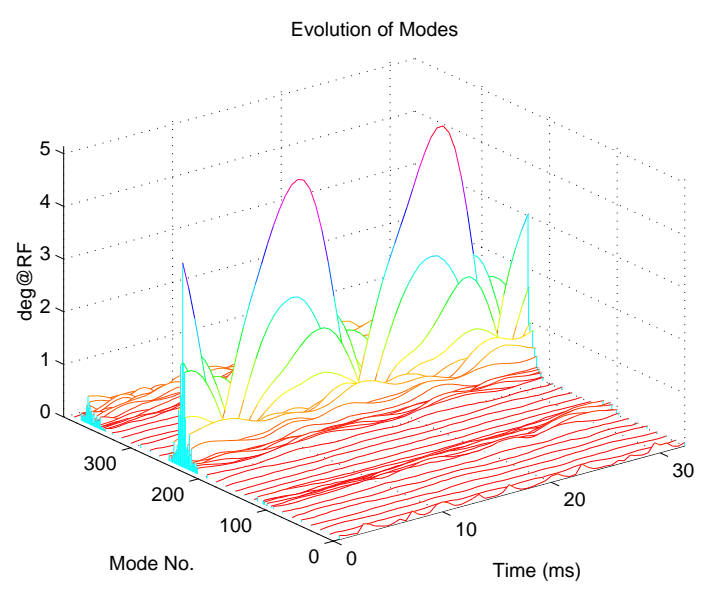

Figure 3: Longitudinal oscillation amplitude versus mode number and time at $280 \mathrm{~mA}$, feedback loop open.

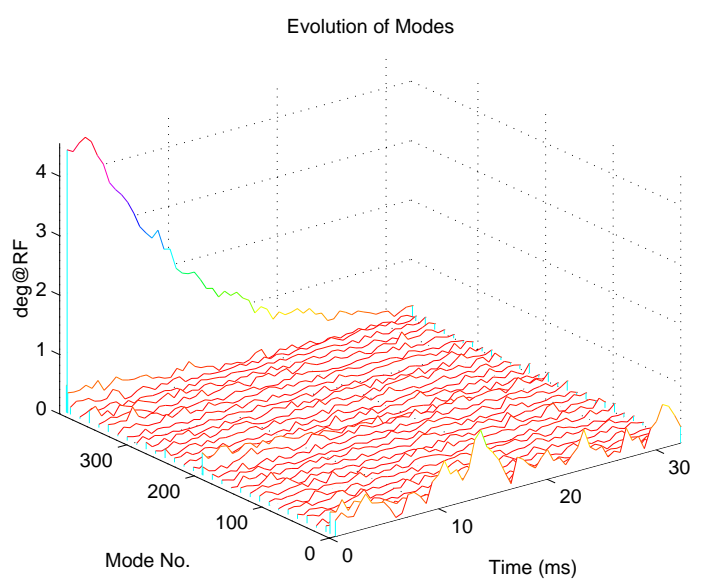

Figure 4: Longitudinal oscillation amplitude versus mode number and time at $15 \mathrm{~mA}$. The anti-damping feedback loop was opened at $t=2 \mathrm{~ms}$.

\subsection{Transverse Feedback System}

The analog electronics of the TFB is similar to the system employed at the ALS (Berkeley) [7], while the digital control unit uses elements compatible with the BESSY II control system [8]. A stripline kicker, designed at BESSY, combines horizonally and vertically deflecting electrodes in one structure [5].

Figure 2 shows the TFB layout. Signals from two BPMs being $\sim 72$ degrees in phase advance apart are used. The horizontal and vertical dipole moments (displacement-charge) are detected at $3 \mathrm{GHz}$, downconverted to baseband by the receivers in heterodyne mode, and combined to produce a correction signal. DC signal components are suppressed by a notch filter that subtracts the correction signals of subsequent revolutions. For each transverse dimension, a $150 \mathrm{~W}$ power amplifier drives a kicker electrode with the opposite electrode floating.

\section{FEEDBACK OPERATION WITH 3RD-HARMONIC CAVITIES}

The presence of four 3rd-harmonic cavities in the storage ring may impair the performance of both feedback systems:

1) HOMs of the 3rd-harmonic cavities are presently the main cause of longitudinal instabilities. Due to thermal drifts, the HOM frequencies depend on the beam current.

2) Passive harmonic cavities cause large variations of the synchronous phase. Detection of longitudinal and transverse bunch position at $3 \mathrm{GHz}$ fails for phase variations exceeding \pm 15 (at $f_{\mathrm{rf}}=500 \mathrm{MHz}$ ). For the LFB, extending the A/D-converter range to accommodate large synchronous phase transients increases the digitization error.

3) Harmonic cavities increase the bunch length by flattening the rf potential. The resulting shift and spread of synchrotron tune may exceed the bandwidth of the 6-tap FIR filter presently used.

Thus, careful tuning of the harmonic cavities with two independent plungers is required: HOMs are tuned to stay away from revolution harmonics for user operation at 150 $250 \mathrm{~mA}$. The ground state is tuned such that a significant lifetime improvement (factor $\sim 1.5$ ) is achieved with moderate phase transients and synchrotron tune shifts. The range of phase transients increases to first order linearly with current and length of the bunch gap [2]. Changing the filling scheme from $2 \times 120$ bunches to one train of 360 bunches followed by a gap of 40 buckets helped to reduce the phase transients while further improving the Touschek lifetime.

Nevertheless, to allow for a larger margin in view of future operation at higher beam current and more extensive bunch lengthening, several modifications are in progress:

A new design of 3rd-harmonic cavities is underway to reduce the HOM Q-values with damping antennas.

For the TFB, a homodyne detector has been built, similar to the detector used at the ALS [9]. Here, instead of an rflocked 3-GHz signal, the reference signal is deduced from each bunch and therefore intrinsically phase locked to the beam. In order to keep this signal independent of the stored current, a controller circuit regulates a PIN-diode attenuator, which operates with little phase shift at the desired frequency. The detector is currently being tested and shows the expected performance so far.

In order to control instabilities in a wider range of synchrotron frequencies, a IIR-filter has been designed and implemented in the LFB [10].

\section{THE LFB AS DIAGNOSTICS TOOL}

Due to its ability to transfer data with or without interrupting feedback operation, the LFB is a valuable diagnostics tool. Examples from several installations of the system are shown in [11]. For BESSY II, the spectral information from Fourier-transformed LFB data is discussed in [12]. Figure 3 shows an example, where an instability centered at mode no. 233 (corresponding to a HOM of a 3rd har- 

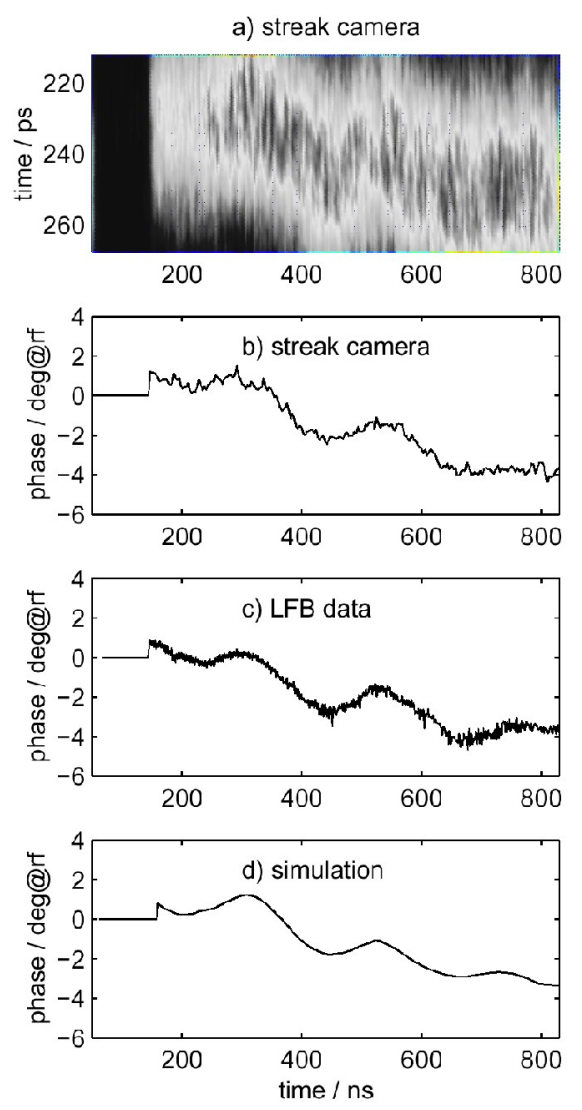

Figure 5: Synchronous phase transients at $200 \mathrm{~mA}$ with a bunch gap of $50 \mathrm{~ns}$. (a) Streak camera image (b) Bunch centroids from the streak camera image (c) Longitudinal feedback data (d) Numerical simulation.

monic cavity at $2.29 \mathrm{GeV}$ ) exhibits a strong beating behavior. As the beam current decays, the beating decreases in amplitude and frequency.

Figure 4 shows the decay of a mode excited before $t=2 \mathrm{~ms}$ by negative feedback. Extrapolating the currentdependent decay times from measurements at $15-50 \mathrm{~mA}$ down to zero current yields $7.9 \pm 0.5 \mathrm{~ms}$, consistent with the expected longitudinal damping time of $7.7 \mathrm{~ms}$.

Synchronous phase transients have been studied in some detail using the LFB as well as a streak camera [13]. A typical situation at $200 \mathrm{~mA}$ with two 3rd harmonic cavities at $3 f_{\mathrm{rf}}+0.3 f_{\circ}$ and two cavities "parked" at $3 f_{\mathrm{rf}}-3.5 f_{\circ}$ is shown in figure 5 ( $f_{\circ}=1.25 \mathrm{MHz}$ is the revolution frequency). The bunch centroids (b) were extracted from the streak camera image (a).

For simultaneously recorded LFB moment signals (current-phase), the rf noise common to all bunches can be used to extract bunch current and synchronous phase [14], the latter shown in (c). Both measurements agree in a peakpeak phase variation of 5 degrees.

Finally, using the LFB bunch currents and measured frequencies of the 3 rd harmonic fundamental modes, a numerical simulation (d) reproduces the main features of the measured phase transients. The modulation on the slope is due to the parked cavities. Rather than computing the full bunch dynamics, the algorithm varies the synchronous phases until the cavity voltage experienced by each bunch matches the synchrotron radiation losses - which characterizes the equilibrium position.

\section{ACKNOWLEDGEMENTS}

The help of our colleagues at SLAC, ALS, INFN and BESSY in setting up, maintaining and improving both feedback systems is gratefully acknowledged.

\section{REFERENCES}

[1] D. Krämer, "BESSY II: Exceeding Design Parameters the First Year of User Service”, EPAC00, Vienna (2000), 640.

[2] S. Khan et al., "Commissioning of the BESSY-II Longitudinal Feedback System”, EPAC00, Vienna (2000), 1903.

[3] T. Knuth et al., "Commissioning Results of the Transverse Feedback System at BESSY II”, EPAC00, Vienna (2000), 1912.

[4] R. Boni et al., "A Waveguide Overloaded Cavity as Longitudinal Kicker for the DA $\Phi$ NE Bunch-by-Bunch Feedback System", Part. Acc. Vol. 52 (1996), 95.

[5] T. Knuth, S. Khan, "Longitudinal and Transverse Feedback Kickers for the BESSY II Storage Ring”, PAC99, New York (1999), 1147.

[6] D. Teytelman et al., "Operation and Performance of the PEP-II Prototype Longitudinal Damping System at the ALS”, PAC95, Dallas (1995), 2420.

[7] W. Barry et al. "Transverse Coupled Bunch Feedback in the Advanced Light Source (ALS)”, EPAC94, London (1994), 122.

[8] J. Bergl et al., "Embedded Controllers, Field Bus and a Modular Concept: Central Elements of BESSY II Controls", PAC97, Vancouver (1997), 2493.

[9] G. Stover (ALS) priv. communication (2000).

[10] D. Teytelman, "Design and Implementation of IIR Algorithms for Control of Longitudinal Coupled-Bunch Instabilities", SLAC-PUB-8411 (2000), and D. Teytelman, priv. communication (2000).

[11] J. Fox et al., "Multi-Bunch Instability Diagnostics via Digital Feedback Systems at PEP-II, DA $\Phi$ NE, ALS and SPEAR", PAC99, New York (1999), 636.

[12] S. Khan, "Spectral Analysis of Coupled-Bunch Instabilities in the BESSY-II Storage Ring”, EPAC00, Vienna (2000), 1136.

[13] Hamamatsu C4742-95. Measurements performed by W. Anders (BESSY), J. Jacob and V. Serriere (ESRF).

[14] S. Prabhakar, "New Diagnostics and Cures for CoupledBunch Instabilities", Ph.D. thesis, SLAC Rep. 554 (2000). 